\title{
Kombipräparat erleichtert die Therapie
}

— Bei fast jedem über 65-Jährigen lassen sich Anzeichen einer Arthrose feststellen. „Mit zunehmendem Lebensalter steigen aber auch Häufigkeit und Anzahl gleichzeitig auftretender Erkrankungen und damit zwangsläufig die Menge der Medikamente, die ein Patient benötigt", führte Dr. Frank Behrens, Centrum für innovative Diagnostik und Therapie, Rheumatologie/Immunologie der Universitätsklinik Frankfurt/M., aus.

Compliance leidet bei hoher Tablettenzahl Der Rheumatologe erinnerte daran, dass die Therapieadhärenz bekanntlich mit der steigenden Anzahl der Medikamente, die ein Patient dauerhaft einnehmen muss, abnimmt. „Aus diesem Grund sind wir als Ärzte daran interessiert, die Tablettenzahl der Patienten nach Möglichkeit zu reduzieren", so Behrens.

Einen wichtigen Beitrag zur Verringerung der Zahl der einzunehmenden Medikamente könnten Kombinationspräparate wie Vimovo ${ }^{\mathrm{TM}}$ leisten, betonte der Rheuma- tologe. Die Fixkombination aus dem nicht steroidalen Antirheumatikum (NSAR) Naproxen $(500 \mathrm{mg}$ ) und dem Protonenpumpeninhibitor (PPI) Esomeprazol $(20 \mathrm{mg}$ ) ist zur symptomatischen Behandlung von Patienten mit Arthrose, rheumatoider Arthritis und ankylosierender Spondylitis zugelassen, die ein erhöhtes Risiko für gastrointestinale Komplikationen aufweisen.

\section{Integrierter PPI-Magenschutz}

Naproxen sei als Bestandteil der Fixkombination ausgewählt worden, weil es nicht nur sehr gut analgetisch wirksam ist, sondern im Vergleich zu allen anderen NSAR auch das geringste kardiovaskuläre Risiko aufweist, erläuterte Behrens. Die Zugabe des PPI Esomeprazol macht die Arthroseschmerztherapie für die Risikopatienten sicherer: In Zulassungsstudien wurde gezeigt, dass das Kombinationspräparat im Vergleich zur Monosubstanz Naproxen die Inzidenz von Magenulzera signifikant reduziert (-76\%; $p<0,001$ [Goldstein JL et al. Aliment Pharmacol Ther 2010;32:

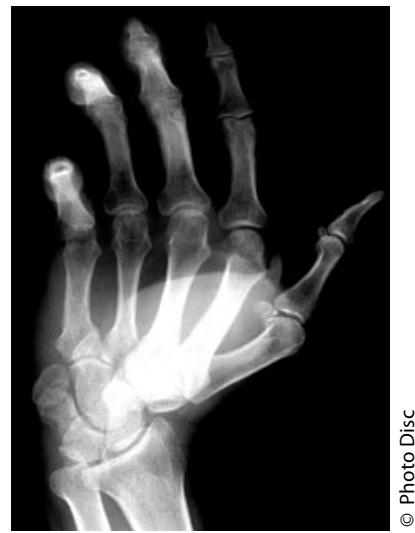

Dieser Patient braucht dauerhaft Schmerzmittel: rheumatoide Arthritis, vor allem der Fingergrundgelenke.

401-413]). „Die Fixkombination ermöglicht Arthrosepatienten somit eine effektive, sichere und zugleich einfache Schmerztherapie. Darüber hinaus kann sie möglicherweise auch zu einer Verbesserung der Therapieadhärenz beitragen", resümierte Behrens.

Dr. Silke Wedekind .

- Quelle: Satellitensymposium „Der multimorbide Patient in der Praxis - eine interdisziplinäre Betrachtung" im Rahmen des 119. Kongresses der Deutschen Gesellschaft für Innere Medizin e. V. (DGIM); Wiesbaden, April 2013 (Veranstalter: AstraZeneca)

\section{Ejaculatio praecox und erektile Dysfunktion}

\section{Sexualstörungen können kombiniert behandelt werden}

- Dass die Ejaculatio praecox (PE) und erektile Dysfunktion (ED) gemeinsam auftreten, ist angesichts der hohen Prävalenz - jeweils rund $20 \%$ - keine Seltenheit. In diesen Fällen bedarf es einer PE-Therapie,

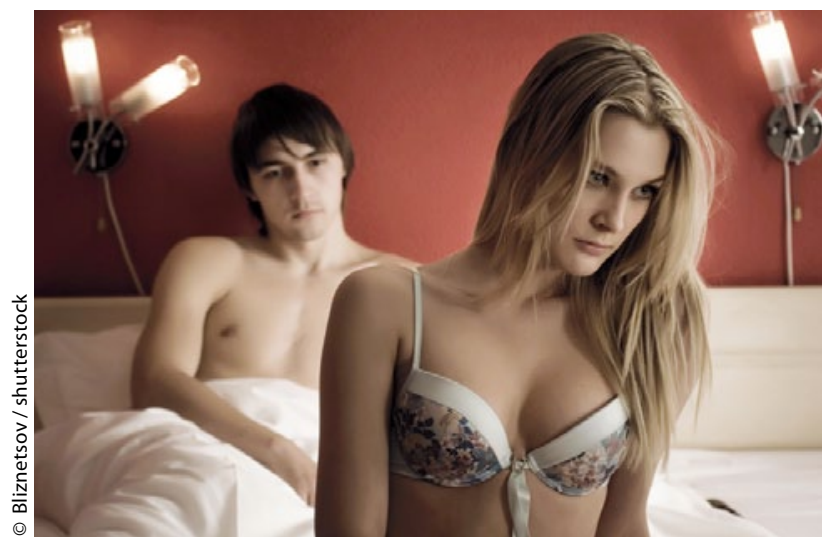

Frust im Schlafzimmer muss nicht sein. die sich mit der Einnahme von PDE-5-Inhibitoren verträgt.

Da Serotonin ist der wichtigste inhibitorische Neurotransmitter in der Kontrolle der Ejakulation ist, erläuterte Prof. Dr. med. Chris McMahon, Universität Sydney, erscheint es logisch, hier mit einem Serotonin-Wiederaufnahmehemmer wie Dapoxetin (Priligy $\left.{ }^{\circledR}\right)$ anzusetzen.

McMahon berichtete über seine Studie, in der 495 Männer, die bereits wegen Potenzstörungen einen PDE5-Inhibitor erhielten und über vorzeitige Ejakulationen klagten, zusätzlich bedarfsweise mit entweder 30 oder $60 \mathrm{mg}$ Dapoxetin oder Placebo versorgt wurden. Auch in dieser Studie bestätigte sich, dass der Therapieerfolg von Dapoxetin sofort einsetzt: Schon ab der ersten Woche zeigte sich ein signifikanter Unterschied zwischen den beiden Gruppen $(p<0,001)$, der bei Studienende nach zwölf Wochen fast zwei Minuten betrug (Ejakulationslatenzzeit 3,4 Minuten unter Placebo, 5,1 Minuten unter Dapoxetin). Parallel dazu verbesserten sich die Ergebnisse in den Domänen „Ejakulationskontrolle“, „Persönlicher Distress" und "Zufriedenheit" des Premature Ejaculation Profile.

Manuela Arand .

- Quelle: Satellitensymposium "Premature Ejaculation and Men's/Couple's Sexual Health", gemeinsamer Kongress der International and European Societies for Sexual Medicine, Berlin, Juni 2013 (Veranstalter: Berlin-Chemie AG/Menarini) 